he use of in vitro release testing as a QA tool to ensure batch-to-batch uniformity has been gaining both increasing popularity and contempt in the pharmaceutical industry. Regardless of views and opinions, it is worthwhile to be prepared to conduct such studies if necessary. Currently, the most publicized methods use horizontal ("Franz or Modified Franz") diffusion cells, with an artificial support membrane to conduct in vitro release studies on a semisolid formulations, typically creams. However, depending upon the type of formulation to be tested, and possible needs for automation, it may be desirable to investigate alternate apparatuses and techniques. The following article gives examples of several techniques and apparatuses which could be employed in release testing of semisolid drug products. which do not impede the free diffusion of drug from the formulation. Using a membrane which is not resistant to the free diffusion of drug allows for the determination of release of drug from the semisolid formulation itself. In addition, it is very important to choose receptor media which maintain adequate sink conditions. Poor sink conditions and/or mixing will result in a large hydrodynamic boundary layer, which will both decrease the amount of drug released, and significantly lower the test's ability to determine differences between formulations. The "goal" in the development of such methods is to have a test which measures the "intrinsic" release of drug from the formulation. In such a system, alterations in the formulations could be measured.

\title{
Techniques For Conducting In Vitro Release Studies On Semisolid Formulations
}

\section{Michael Corbo, Ph.D}

\section{Method Development}

The actual development of in vitro release methods has been discussed in the past, and will not be addressed in detail here ${ }^{1}$. However, it is important to reiterate the need to use artificial support membranes

\section{Formulations Considerations}

A primary concern in the selection of an in vitro release technique is the nature of the formulation to be tested. The type of formulations which are typically tested fall into the broad classifications of: creams,

\section{Figure 1}

Franz Diffusion Cell

\section{SAMPUNG}

PORT

\section{TOP PLATE}

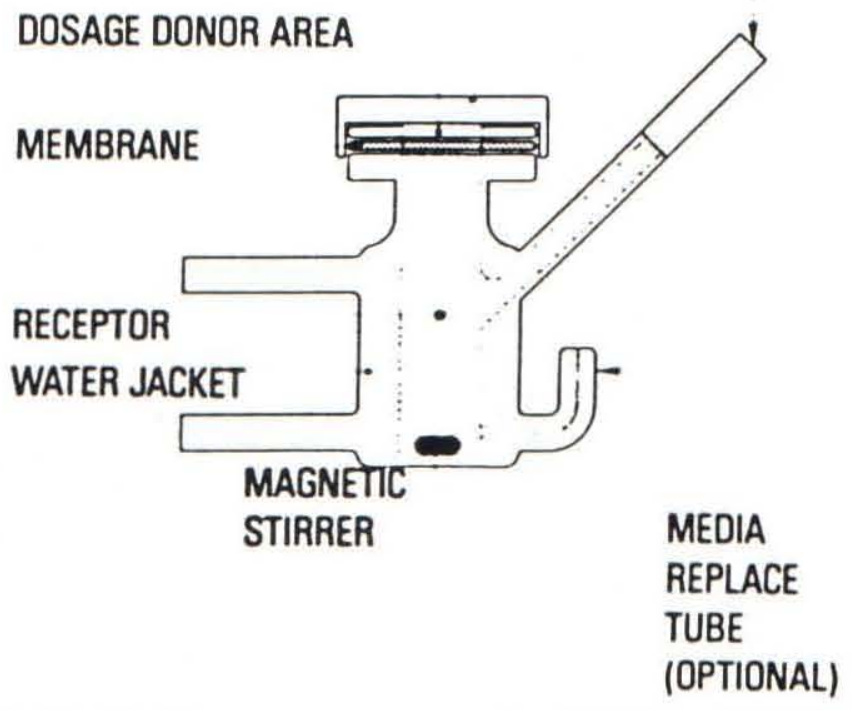

gels, lotions/shampoos, and ointments. Creams and gels have been tested using the Franz cell technology, which works quite well. Lotions and shampoos, while less viscous than creams and gels, typically can be tested using similar techniques, with careful selection of artificial support membranes. For instance, a cream or gel formulation could be tested using a $70 \mu \mathrm{m}$ mesh membrane, while a lotion would simply run right through such a large pore size. In general, ointments are the most difficult formulations to conduct in vitro release studies upon. With Franz Cell techniques, ointments usually demonstrate extremely low release rates.

\section{Common Techniques}

As indicated earlier, the most popular technique for conducting in vitro release studies is the use of horizontal "Franz" diffusion cell technology. In such studies, (see Figure 1) a receptor solution is placed into the receptor compartment, 


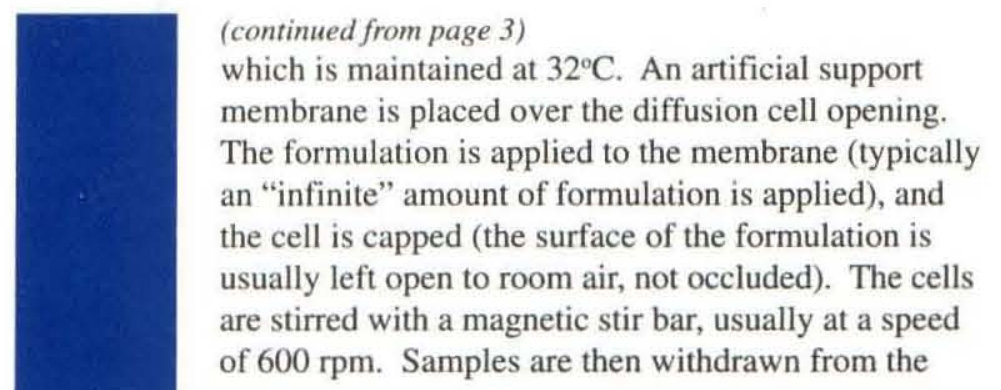

(continued from page 3 )

which is maintained at $32^{\circ} \mathrm{C}$. An artificial support membrane is placed over the diffusion cell opening. The formulation is applied to the membrane (typically an "infinite" amount of formulation is applied), and the cell is capped (the surface of the formulation is usually left open to room air, not occluded). The cells of $600 \mathrm{rpm}$. Samples are then withdrawn from the measuring dissolution rather than release. However, for formulations which do not dissolve/disperse in the receptor, this technique could be used to conduct in vitro release studies, using well-established, universal equipment. In addition, such a technique is amenable to full automation using robotics.

Van Kel recently introduced the Enhancer Cell, which could be used for in vitro release testing. The Enhancer Cell technique is very similar to the

\section{Techniques For Conducting In Vitro Release Studies On Semisolid Formulations}

paddle-over-disk method (See Figure $2)$, where the Enhancer Cell rests in the bottom of a dissolution vessel, with a paddle stirring media above it. The main difference in the

receptor solution at regular time intervals, typically over the course of 6-8 hours.

While this technique has been proven to be quite useful, it tends to be a rather complicated process, which requires extensive training to generate reproducible results. In addition, the method does not lend itself easily to full automation (i.e. having samples


prepared and letting a robot set up and conduct the study). These are relatively new apparatuses in the area of QA testing, and there are several standardization points which are currently being addressed: these include; there is no standard diffusion cell, they vary widely in size (volume and surface area) and style; there is no accepted in-use calibration procedure at this point; and there is no standard size stir-bar. Another technique which has been used is the USP paddle-over disk method. In such studies, the formulation is filled into a USP stainless steel disk, placed on the bottom of a dissolution vessel which is filled with receptor media, and then stirred (paddles, $25 \mathrm{~mm}$ over the disk) at $50 \mathrm{rpm}$. This technique does not use a membrane (the only barrier is the stainless steel mesh on the disk). One major draw-back of this technique is the potential for the formulation to dissolve in the receptor media, thus techniques, is the ability to use artificial membranes with the Enhancer Cell. The use of an artificial membrane can significantly reduce or eliminate problems associated with dissolution/dispersion of the formulation. As with paddle-over-disk, this technique lends itself to full automation.

Another technique which can be used is the inverted rotating cylinder (Figure 3 ). This technique simply uses a machined, round cavity in a piece of Teflon or stainless steel as the "holder" of the drug product. Once loaded with formulation, the cylinder can be lowered into a standard USP dissolution vessel, and rotated (typically at $50 \mathrm{rpm}$ ). This method does not use a membrane, and can only be used with formulation which do not dissolve or disperse in the receptor medium. This method has been shown to be quite useful for hydrophobic ointment bases containing solubilized drug. Once again, this technique can be fully automated.

\section{Comparison of Techniques For Cream And Ointment F ormulations}

Examples of the use of the above techniques will be presented for a cream formulation (Terazol 3 Vaginal Cream, $0.8 \%$ terconazole, drug is in suspension) and an ointment (petrolatum based containing a soluble highly lipophilic drug). These two formulations represent the extremes in formulations, where the release of terconazole is quite rapid into an aqueous buffer, and the release of the active from the ointment is extremely slow into a hydro-alcoholic medium. Both formulations were tested using the above techniques to demonstrate the utility of each method for the formulations. All data is normalized for surface area. In all studies, samples were taken at $1,2,4$ and 6 hours.

\section{In Vitro Release Of Terconazole:}

For all studies conducted with terconazole, a receptor medium of $\mathrm{pH} 4$ citrate-phosphate buffer was used.

Franz Cell: Modified Franz Diffusion Cells (Crown Glass, Somerville, New Jersey), with a ground- 
glass surface, $9 \mathrm{~mm}$ opening and $10 \mathrm{~mL}$ volume were used. An artificial membrane of a $70 \mu \mathrm{m}$ Teflon Mesh was used.

Paddle-over-Disk: Standard USP disks were filled with $1 \mathrm{~g}$ of formulation and placed in the bottom of a USP dissolution vessel containing $250 \mathrm{~mL}$ of receptor media. Paddles were rotated at $50 \mathrm{rpm}, 25$ $\mathrm{mm}$ above the surface of the disks.

Enhancer Cell: Enhancer cells, with the same $70 \mu \mathrm{m}$ Teflon mesh membrane were used. Studies were conducted in a USP dissolution vessel, containing $250 \mathrm{~mL}$ of receptor media. Paddles were rotated at $50 \mathrm{rpm}, 25 \mathrm{~mm}$ above the surface of the Enhancer Cells.

Inverted Enhancer Cell: Since this O/W formulation would readily disperse into the medium, it was not possible to use the inverted rotating cylinder. However, for comparison, an Enhancer Cell, equipped with the $70 \mu \mathrm{m}$ Teflon mesh membrane, was tapped and inverted into a dissolution vessel containing $250 \mathrm{~mL}$ of receptor media. The apparatus was rotated at $50 \mathrm{rpm}$.

\section{In Vitro Release Of A Lipophilic \\ Drug From An Ointment}

For all studies, a receptor medium of $50 \%$ ethanol in water was used.

Franz cells and Enhancer Cells, as above, were used with $70 \mu \mathrm{m}$ Teflon mesh as the support membrane. Paddle-over disk studies were conducted as above. The inverted rotating cylinder was used for this formulation, since actual dissolution/dispersion of the ointment did not occur in this media.

\section{Results}

\section{Terconazole:}

Results for the in vitro release of terconazole using the various techniques are summarized in Figure 4. Data is presented as cumulative amount released vs the square root of time. Since the terconazole is dispersed throughout the formulation as a solid suspension, the release profile follows the Higuchi Equation $^{2,3}$.

Results indicated that the release of terconazole was similar from the Franz Cell and the Enhancer Cell. Results from the inverted Enhancer Cell were somewhat lower, and more variable. These lower results were most likely due to poor mixing at the surface of the membrane (the apparatus was not designed for this purpose, and mixing at the membrane surface when inverted is poor). Results from the paddle-over-disk were significantly higher than those from the Franz and Enhancer Cells. This increase in release was due to the dispersion of the cream into the receptor medium. When such dispersion occurs, the actual surface area of formulation in contact with the medium increases dramatically, resulting in an apparent increase in the release rate. Results from the paddleover disk method with this formulation demonstrate



that one needs to ensure that formulations should not disperse in the receptor media during a test procedure.

\section{Lipophilic Drug In Ointment Base}

The results for the in vitro release of a lipophilic drug from a hydrophobic ointment are summarized in Figure 5. Results are presented at the cumulative amount released vs time (since the drug is fully dissolved in the ointment, release kinetics would not follow the Higuchi Equation). With extremely hydrophobic compounds, a major problem in approaching the "intrinsic" release of the active is the minimization of the hydrodynamic boundary layer. Careful selection of the receptor solution is of primary importance, however, even the most appropriate receptors can not over-come poor mixing conditions at the formulation/media interface. Results indicated that release was highest from the inverted rotating cylinder, followed by the paddle-over-disk, Enhancer Cell and finally the Franz Cell. Since there was not apparent dispersion of the formulation into the media, differences in the release rates are most likely due to the effect of mixing at the formulation/media interface. For instance, mixing is most efficient in the inverted rotating cylinder, resulting in a lower hydrodynamic boundary layer, thereby increasing the apparent release rate. Other studies conducted at higher stirring rates suggested that the inverted rotating cylinder apparatus was approaching the intrinsic release rate.

\section{Conclusions}

In general, the Franz Diffusion Cell is the current "apparatus of choice" from a regulatory perspective ${ }^{4,5}$. In the development of methods, the Franz Cell is usually the first apparatus to be investigated. However, when the formulation (i.e. lipophilic ointments) or the necessity for full automation precludes the use of the Franz Cell, it may be

(continued on page 6)

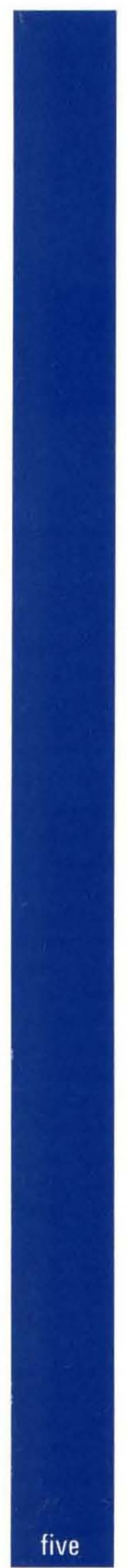




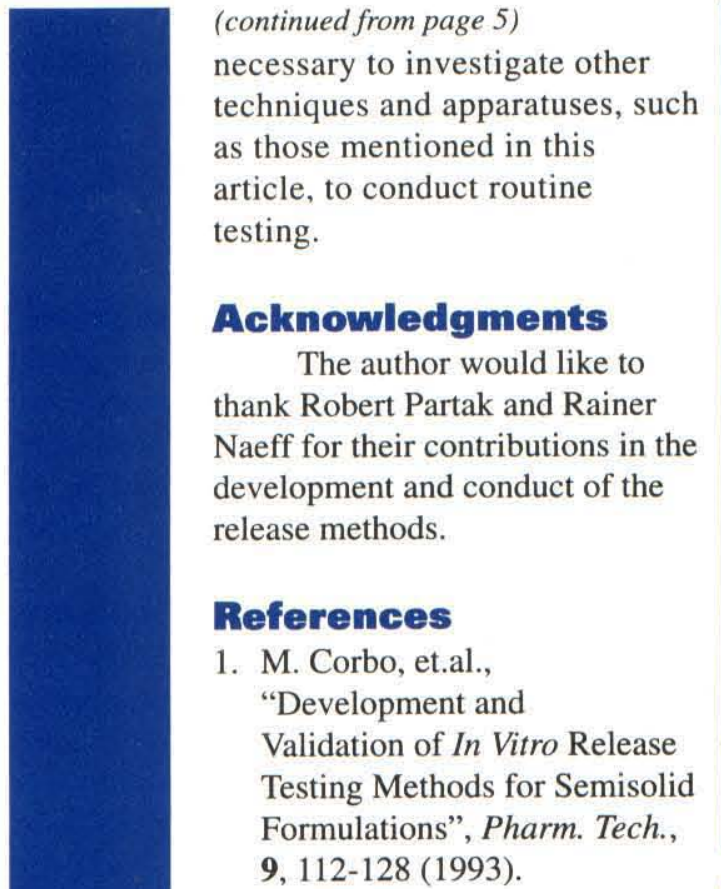

2. T. Higuchi, "Sustained release medications", J. Pharm. Sci. 52 1145-1148 (1963).

3. R.H. Guy and J Hadgraft, "On the determination of drug release rates from topical dosage forms", Int. J. Pharm., 60 R1-R3 (1990).

4. V.P. Shah, J. Elkins, S.-Y. Lam and J.P. Skelly, "Determination of in vitro drug release from hydrocortisone creams", Int. J. Pharm. 53 53-59 (1989).

5. V.P. Shah, J. Elkins, J. Hanus, C. Noorizadeh and J.P. Skelly, "In vitro release of hydro cortisone from topical preparations and automated procedure", Pharm. Res. 8 55-59 (1991).

\section{Figure 4}

In Vitro Release of Terconazole using Various Techniques

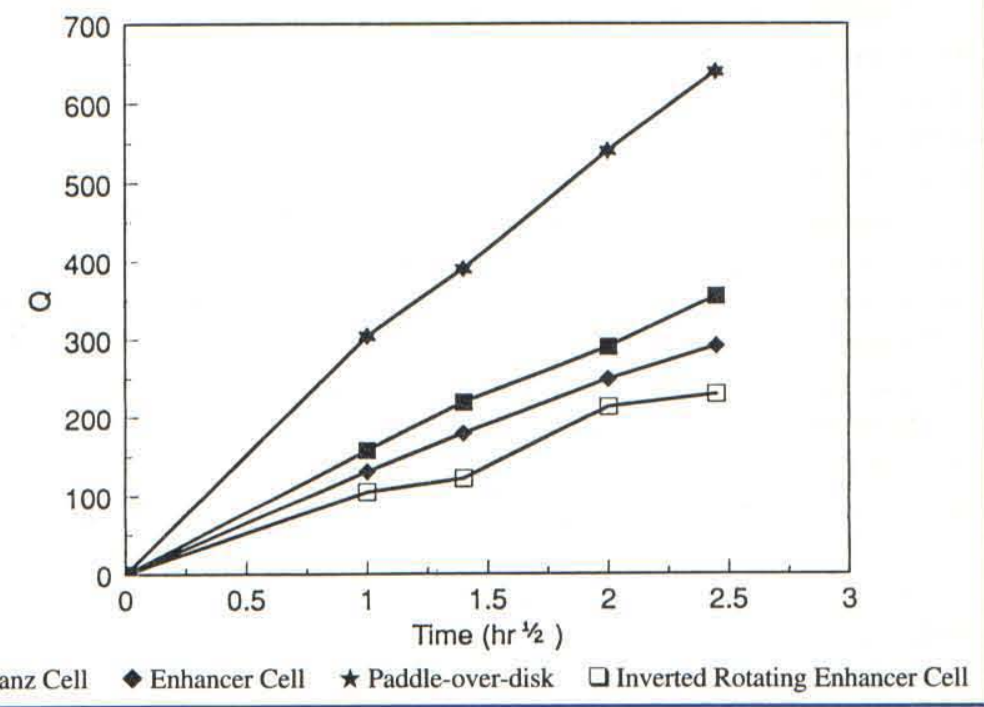

\section{Figure 5}

In Vitro Release of a Lipophilic Compound from an Ointment

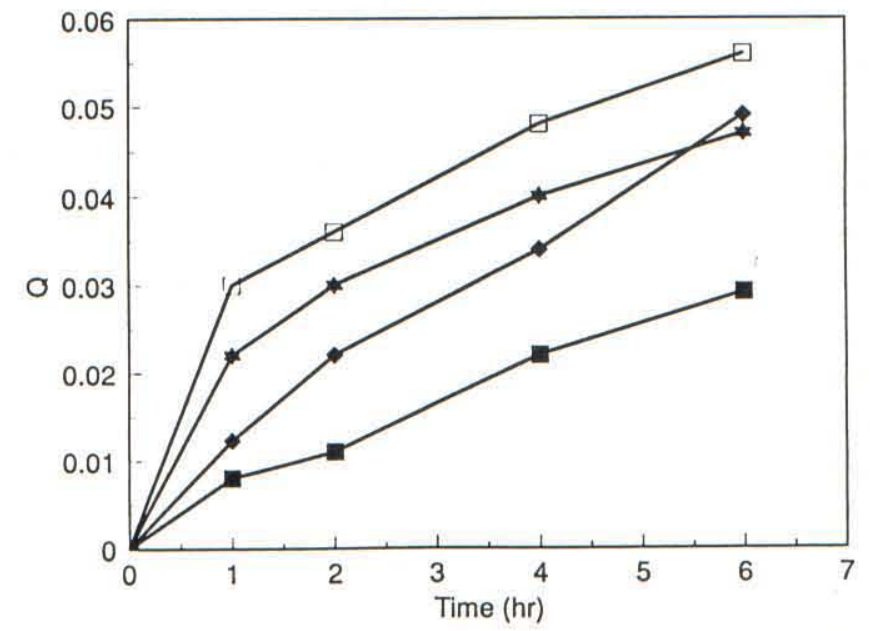

Franz Cell Enhancer Cell $\star$ Paddle-over-disk $\square$ Inverted Rotating Cylinder 